\title{
Feasibility Study To Assess The Delivery of A Novel Isometric Exercise Intervention For People With Stage 1 Hypertension In The NHS: Protocol For The Isofit-BP Study Including Amendments To Mitigate The Risk of COVID-19
}

Jonathan Wiles ( $\square$ jim.wiles@canterbury.ac.uk)

University of Kent https://orcid.org/0000-0002-7790-8063

Melanie Rees-Roberts

University of Kent

Jamie O'Driscoll

Canterbury Christ Church University

Timothy Doulton

East Kent Hospitals NHS Trust: East Kent Hospitals University NHS Foundation Trust

Douglas Mclnnes

Canterbury Christ Church University

Vanessa Short

NHS Canterbury and Coastal CCG

Tracy Pellatt-Higgins

University of Kent

Katie Saxby

University of Kent

Katerina Gousa

University of Kent

Alan West

Medway NHS Foundation Trust

Maggie Smith

University of Kent

Ellie Santer

Canterbury Christ Church University https://orcid.org/0000-0002-5437-0045

John Darby

University of Kent

Chris K Farmer

University of Kent 


\section{Study Protocol}

Keywords: Isometric exercise, exercise, hypertension, general practice, feasibility study, COVID-19

Posted Date: January 19th, 2021

DOl: https://doi.org/10.21203/rs.3.rs-149399/v1

License: (c) (i) This work is licensed under a Creative Commons Attribution 4.0 International License. Read Full License 


\section{Abstract}

Background: Hypertension (HTN) affects approximately $25 \%$ of the UK population and is a leading cause of mortality. Associated annual health care costs run into billions. National treatment guidance includes initial lifestyle advice, followed by anti-hypertensive medication if blood pressure (BP) remains high. However, adoption and adherence to recommended exercise guidelines, dietary advice and antihypertensive medication is poor. Four short bouts of isometric exercise (IE) performed 3 days per week (d/wk) at home elicits clinically significant reductions in BP in those with normal to high-normal BP. This study will determine the feasibility of delivering personalised IE to patients with Stage 1 hypertension for whom lifestyle changes would be recommended before medication within NHS primary care.

Methods: This is a randomised controlled feasibility study. Participants $18+$ years, with Stage 1 hypertension, not on anti-hypertensive medication and without significant medical contraindications. Trial arms will be standard lifestyle advice (control) or isometric wall squat exercise and standard lifestyle advice. Primary outcomes include success of intervention delivery and change in BP. Secondary outcomes include accuracy of protocol delivery, execution of and adherence to protocol, recruitment rate, attrition, perception of intervention viability, cost, participant experience, and accuracy of home BP. The study will last 18 months. Sample size of 100 participants (50 per arm) allows for $20 \%$ attrition and $6.5 \%$ incomplete data, based upon 74 (37 each arm) participants (two-sided 95\% confidence interval, width of 1.33 and standard deviation of 4) completing 4 weeks. Ethical approval IRAS ID: 274676.

Discussion: Before the efficacy of this novel intervention to treat Stage 1 hypertension can be investigated in any large randomised controlled trial, it is necessary to ascertain if it can be delivered and carried out in a NHS primary care setting. Findings could support IE viability as a prophylactic / alternative treatment option.

Trial Registration: International Standard Randomised Controlled Trial Number (ISRCTN) 13472393 registered 18 August 2020. http://www.isrctn.com/ISRCTN13472393

\section{Introduction}

\section{Background and rationale $\{6 a\}$}

Hypertension ( $\geq 140 / 90 \mathrm{mmHg}$ ) (1) affects approximately 1 in 4 people in the UK and is a leading modifiable risk factor for mortality (2). Furthermore, estimates indicate that the annual burden from conditions attributable to hypertension is over $£ 2$ billion (3) in England, with long-term care following a debilitating heart attack, stroke and/or vascular dementia often precipitated by hypertension costing substantially more (4).

\section{Standard care for hypertension}


National guidance for the treatment of hypertension is graded from lifestyle intervention (i.e. advice about diet, weight management, exercise, alcohol intake) to pharmacological therapy depending upon severity and duration of hypertension when other factors have been taken into account such as age, co-morbidity and the presence of target organ damage (1). The goal of antihypertensive therapy is generally to reduce clinic BP to $<140 / 90 \mathrm{mmHg}$, although recommended targets vary depending on age and co-morbidity (1). However, up to $50 \%$ of people fail to achieve their target BP (5) mainly due to non-compliance (estimated $30 \%-50 \%$ failing to comply at 6 and 12 months, respectively) (6) with undesirable side effects of antihypertensive medication often cited in this context (7).

\section{Lifestyle interventions}

The importance of lifestyle changes to aspects, such as diet and exercise habits for patients with hypertension in the absence of other risk factors should not be overlooked (1). Additional treatment options are also in keeping with the UK government's commitment to provide greater patient choice (8). Whilst dietary strategies can be effective, these are difficult to fully adopt and maintain (9).

Evidence suggests that exercise may be as effective as medication in controlling BP (10-12) and it is often promoted as a treatment option for those with Stage 1 hypertension (defined as a blood pressure of 140-159/90-99 mmHg), without co-morbidity or other long-term conditions (1). Current exercise guidelines for the prevention and treatment of hypertension recommend that adults accumulate a mainstay of $30+$ minutes of moderate intensity aerobic exercise on 5 , but preferably all, days per week (d/wk), supplemented by moderate to vigorous resistance exercise $2-3 d / w k$ and general flexibility exercise $\geq 2-3 \mathrm{~d} / \mathrm{wk}$ (13). However, in a randomised controlled trial, a long-term programme of aerobic exercise training failed to reduce clinic BP (14). This may be due to poor adherence $(67 \%)$ to the relatively high amounts of aerobic exercise recommended (14). Furthermore, other studies have demonstrated attrition rates as high as $50 \%$ during traditional aerobic exercise interventions (15). Indeed, evidence typically suggests that a significant challenge are the low adoption and high attrition rates associated with these guidelines (16). Thus, effective and manageable lifestyle interventions with respect to exercise remain an unmet clinical need. It is suggested that to promote lifestyle exercise changes, patients need easily adopted, effective and manageable exercise interventions as a first line option for managing their BP.

\section{Isometric exercise}

Meta-analyses indicate that isometric exercise (IE) results in larger reductions in BP when compared with either aerobic and dynamic resistance exercise training (11) and has great potential to treat hypertension (17), improve patient health and reduce mortality risk. We have shown that IE can lower BP in people with both normal (18) and high-normal (pre-hypertensive) (19) BP and therefore has the potential to be an effective lifestyle intervention for hypertension. IE involves holding a fixed position for a period of time; skeletal muscles are used but there is no movement, e.g. the wall squat which involves leaning against a wall in a seated position (Fig. 1). Moreover, only 24 minutes of isometric wall squat exercise a week are 
required to achieve reductions in $\mathrm{BP}$ of $12 / 6 \mathrm{mmHg}$ in pre-hypertensives, which can be easily carried out at home without the need for costly equipment (19).

\section{Contextualising current findings}

The importance of these findings is substantial considering a $10 \mathrm{mmHg}$ reduction in systolic BP and $5 \mathrm{mmHg}$ reduction in diastolic BP is associated with a $40 \%$ lower risk of stroke and $30 \%$ lower risk of mortality from heart disease and other vascular causes throughout middle age (20). Although we have consistently demonstrated that IE can lower BP $(19,21-23)$, interpretation of the results, along with the findings of others is limited by small participant numbers $(18-19,23-29)$. Isometric exercise may provide a new viable solution with respect to exercise for those with Stage 1 hypertension, evidence for the efficacy of IE in this clinical population is still not robust. Furthermore, this intervention has never been tested within a NHS setting, nor confirmed in any large randomised control trials.

\section{Study rationale}

As the most common long-term health condition in the UK and a primary risk factor for mortality, hypertension is a serious health problem (3). With every $20 \mathrm{mmHg}$ increase in systolic BP above $115 \mathrm{mmHg}$ and $10 \mathrm{mmHg}$ increase in diastolic BP above $75 \mathrm{mmHg}$, the risk of death from cardiovascular disease doubles (30). Public Health England (PHE) suggests that there is an opportunity to prevent more than 9,000 heart attacks and at least 14,000 strokes over three years with better detection and management of high $\mathrm{BP}$, high cholesterol and atrial fibrillation (20). It has been further estimated that over ten years, 45,000 quality adjusted life years and $£ 850 \mathrm{~m}$ could be saved if England achieved a $5 \mathrm{mmHg}$ reduction in population systolic BP (3). In 2007, the NHS and PHE announced a drive to prevent thousands of heart attacks and strokes but highlighted the need for research into innovative lifestyle modifications to lower BP (3). New personalised support for lifestyle changes, like our IE training prescription, delivered by primary care allied health professionals could support this drive without directly adding to GP workload.

Research suggests that IE can lower resting BP in people with hypertension enough to effect a clinically significant reduction in cardiovascular disease and all-cause mortality (19). Isometric exercise offers distinct advantages over other forms of exercise (17) making it a better lifestyle treatment for hypertension. A frequently cited barrier to exercise is lack of time (31). Though aerobic exercise guidelines recommend $\geq 150$ minutes per week (32), it has been demonstrated that adherence is better with shorter bouts of exercise (33). Our evidence-based IE wall squat programme can be easily prescribed by health care providers and is very simple to execute regardless of age or physical ability. It does not require costly equipment or access to specialist facilities, does not require specific clothing, and most importantly, is easily performed at home. Empowering patients to manage their condition is key and use of short, simple, personalised exercise that can be carried out at home may enable this. Furthermore, our personalised exercise 'prescription' ensures optimal IE intensity is achieved, helping to improve confidence, motivation and patient adherence $(31,34)$. 


\section{Objectives $\{7\}$}

The aim of the study is to determine the feasibility of delivering an individually tailored IE training programme to patients with Stage 1 hypertension (defined as a clinic BP of $140-159 / 90-99 \mathrm{mmHg}$ ) for whom lifestyle changes would be recommended before pharmacological treatment within a primary care NHS setting. Furthermore, the primary objectives are as follows:

1. To assess if nurses/allied health professionals (e.g. health trainer, healthcare assistants, physiotherapists) can deliver isometric exercise prescriptions for Stage 1 hypertensive patients in a primary care NHS setting.

2. To estimate the variance in BP change, to enable sample size calculation for a definitive randomised controlled trial.

The secondary objectives are as follows:

1. Evidence the fidelity of the study intervention with respect to healthcare professional delivery and patient completion of IE.

2. Estimate short- (4-week) and medium-term (3-and 6-month) adherence rates to IE intervention.

3. Estimate recruitment and attrition rates at recruiting GP sites to inform future trials.

4. Explore the willingness of GPs, secondary care clinicians and healthcare professionals to consider IE as a treatment option for patients, including barriers and facilitators for delivering and integrating this within an NHS care pathway for hypertension.

5. Establish the cost and cost-utility of the IE intervention compared to standard care for Stage 1 hypertension.

6. Understand participant experiences of undertaking IE, adherence to the programme and continuation. Additionally, explore possible negative effects of COVID-19 on recruitment rates and participation.

7. To investigate the feasibility of using observed home blood pressure readings for remote monitoring

\section{Trial Design $\{8\}$}

\section{Design}

Multi-centre randomised controlled feasibility study.

\section{Random allocation}

Those that meet the inclusion criteria will be randomised to one of two groups in a 1:1 ratio. One hundred participants will be recruited; therefore, 50 participants will be allocated to each group.

\section{Amendments due to COVID-19 pandemic}

As a result of the unprecedented spread of a novel coronavirus early in 2020, by mid-March the European region had become the epicentre of a COVID-19 pandemic (35). An outcome of this was that many 
recently funded health projects using human participants, including this study, were required to reevaluate their viability and where necessary revise their research design, methods and protocols in line

with general Government and specific funder guidance (e.g. NIHR Restart Framework 2020 ${ }^{36}$ ) to help ensure the continued safety of research participants and personnel alike. The main changes made to this study are documented (identified in italics) to provide the reader with greater insight into how this research project evolved to become fit for purpose as part of what has now become a new research normal.

\section{Methods: Participants, Interventions And Outcomes}

\section{Study setting $\{9\}$}

GP practices in the South East of England.

\section{Eligibility criteria $\{10\}$}

Patients aged 18+ with Stage 1 Hypertension who are not yet on anti-hypertensive medication and without any significant medical condition that would contraindicate their participation.

\section{Exclusion criteria}

Patients who are taking anti-hypertensive medication; have white coat hypertension (as evidenced by averaged home systolic BP $<135 \mathrm{mmHg}$ ); are unable to undertake the study intervention (isometric exercise); have a previous history of diabetes mellitus (Type 1 or type 2), known or suspected ischaemic heart disease (including myocardial infarction and/or angina and/or coronary revascularization procedure), moderate or severe stenotic or regurgitant heart valve disease, atrial or ventricular arrhythmia, stroke or transient ischaemic attack, aortic aneurysm and/or peripheral arterial disease, uncorrected congenital or inherited heart condition; have an estimated glomerular filtration rate $<45 \mathrm{ml} / \mathrm{min}$ (calculated using CKD-EPI or MDRD formulae, and taking most recent documented results); have a documented left ventricular ejection fraction $<45 \%$ and/or left ventricular hypertrophy (by either echocardiography or standard ECG criteria e.g. Sokolow-Lyon); have a documented urine albumin:creatinine ratio $>3.5 \mathrm{mg} / \mathrm{mmol}$; are unable to provide informed consent; are enrolled in another Clinical Trial of an Interventional Medicinal Product or Medical Device or other interventional study; and if female, are pregnant or currently breast feeding. Then finally, any medical condition that, in the opinion of the investigator, would make the participant unsuitable for the study.

\section{Who will take informed consent? $\{26 \mathrm{a}\}$}

Trained healthcare professionals will be obtaining informed consent via discussion on video call and completion via online software or hardcopy. 
Not applicable

\section{Interventions}

\section{Explanation for the choice of comparators $\{6 \mathrm{~b}\}$}

Not applicable

\section{Intervention description $\{11 \mathrm{a}\}$}

Standard care lifestyle advice (Arm 1 - control) or isometric wall squat exercise training as previously described by Wiles et $\mathrm{al}^{37}$ with standard care lifestyle advice (Arm 2). Figure 2 shows flow-chart of selection of participants and interventions.

\section{Criteria for discontinuing or modifying allocated interventions $\{11 \mathrm{~b}\}$}

Modification of the isometric exercise prescription will be made if appropriate, after the participant's first week of exercise training, as part of a fidelity check.

\section{Strategies to improve adherence to interventions $\{11 \mathrm{c}\}$}

Participants will receive reminder text or email messages to help adherence to standard care advice, collecting home blood pressure measurements and isometric exercise training (for Arm 2 participants).

\section{Relevant concomitant care permitted or prohibited during the trial $\{11 \mathrm{~d}\}$}

Exclusion criteria states the participants should not be taking anti-hypertensive medications whilst taking part in the trial.

\section{Provisions for post-trial care $\{30\}$}

The participants in the control Arm will be offered an Isometric exercise intervention after they have completed their time in the study. The GP practice will oversee participant's general care throughout the trial and participants will be returned to the care of their GP after the trial.

\section{Outcomes $\{12\}$}

\section{Primary:}

1. Feasibility of isometric exercise prescription assessed using qualitative data from healthcare professional focus groups at month $11 / 12$ of the study

2. Feasibility of isometric exercise intervention and study assessed using qualitative data from participants at month 7 and 11 of the study

3. Variance of blood pressure changes from baseline using participant blood pressure data at week 4 , months 3 and 6 
4. Sample size for a definitive randomised controlled trial, calculated using evidence of effect on participant systolic blood pressure change at week 1 , months 3 and 6

\section{Secondary:}

1. Fidelity of the isometric exercise prescription measured using prescription competency assessment data at month 3

2. Fidelity of the isometric exercise prescription measured using observation data from the first Incremental Isometric Exercise Test (IIET) delivered from month 3 to month 5

3. Fidelity of the isometric exercise prescription defined by participant heart rate data within HR reference intervals at Day 7-10

4. Short and medium-term adherence rates recorded as those adhering to isometric exercise intervention at week 4, month 3 and month 6

5. Recruitment and attrition rates from data collected at sites at month 10 and month 15

6. GPs and healthcare professionals' attitudes to isometric exercise as a treatment option for patients, measured using remote focus groups and telephone interviews at month 11/12

7. Cost and cost-utility of the isometric exercise intervention using healthcare resource use data and quality-adjusted life years (QALYs) at month 15 (or last patient follow up)

8. Participant experiences of undertaking isometric exercise using participant isometric exercise experience surveys at week 4

9. Effect of COVID-19 on recruitment rates and participation using participant focus groups or telephone calls at month 7 and 11 of the study

10. Feasibility of using observed home blood pressure readings for remote blood pressure monitoring, using participant blood pressure data and observations from the measures at day 1 , week 4 , month 3 and month 6

\section{Participant timeline $\{13\}$}

\section{Sample size $\{14\}$}

Review of current literature revealed few IE studies in a hypertensive population. These studies were small $(\mathrm{n}<25)$, conducted under different conditions to the proposed study, and showed low precision and large variability in estimates of the standard deviation (SD). A sample size of 100 participants, 50 per arm, will be used in the study. Allowing for $20 \%$ attrition and $6.5 \%$ incomplete data, 74 participants (37 in each arm) will have completed change measures at 4 weeks. This is in line with the recommended sample size of 70 to estimate key parameters from external pilot RCTs (38). A sample size of 74 produces a two-sided $95 \%$ confidence interval with a width of 1.33 when the standard deviation is 4 . This estimate of 4 has been taken from a previous study $(\mathrm{N}=24)(19)$. The sample size confidence interval has been calculated using Pass 11 software (PASS 11. NCSS, LLC. Kaysville, Utah, USA. www.ncss.com).

\section{Recruitment \{15\}}


Participants will be identified opportunistically through patient database searches and invited to participate. The study will also be advertised with ethically approved advertising materials, in participating GP practices, with electronic adverts on their websites, social media and newsletters as well as by text message to potential participants.

\section{Assignment of interventions: allocation}

\section{Sequence generation $\{16 a\}$}

Random permuted blocks will be used within stratification (of site and age), ensuring that treatments are balanced at the end of every strata block.

\section{Concealment mechanism $\{16 \mathrm{~b}\}$}

Participants will be allocated to either the control or isometric exercise arm using a third-party supplier of randomisation services (39). This internet-based service allows investigators to randomise patients from anywhere in the world through a web browser.

\section{Implementation $\{16 c\}$}

The investigators will implement the allocation created by the online randomisation software (39).

\section{Assignment of interventions: Blinding}

\section{Who will be blinded $\{17 \mathrm{a}\}$}

Not applicable.

\section{Procedure for unblinding if needed $\{17 b\}$}

Not applicable.

\section{Data collection and management}

\section{Plans for assessment and collection of outcomes $\{18 \mathrm{a}\}$}

The success of intervention delivery by health care professionals in a primary NHS healthcare settings will be determined using both qualitative data (remote) and the heart rate data recorded by the participants at the end of each isometric wall squat bout $x 4$ per session over the 3 sessions completed in the first week using a wireless heart rate monitor and chest strap (Sigma PC 15.11, Neustadt /Weinstraße, Germany). The average HR calculated from the first 3 sessions will then be compared against the individuals' target training HR (calculated as $95 \%$ of HR peak measured during the individuals incremental isometric exercise test IIET) with an acceptable target heart rate range (THRR) of $76-111 \%$ of heart rate peak ${ }^{37}$. Perceptions of intervention delivery by healthcare professionals will be explored in qualitative focus groups (remote). 
Any change in BP following the IE intervention will be determined using the home blood pressure data (measured using an automatic upper arm blood pressure monitor [Omron M3 Intellisense, Kyoto, Japan] at weeks 4, 12 and 24) recorded in the participants diaries and comparing this against their observed baseline (recorded at day 1). This data will also be used to estimate BP variance by calculating the difference in systolic BP change from baseline at each endpoint between the isometric exercise and control group with $80 \%$ and $95 \%$ confidence intervals.

Accuracy of IE protocol delivery will be determined using data from three fidelity assessments; the first being healthcare professional competency checks administered during their isometric exercise prescription training; the second being expert observation /evaluation of at least the first IIET delivered by each HCP; and then finally, by third party examination of the first week of heart rate data recorded in the study diaries of each HCP's specific participants, with average HR calculated from the first 3 sessions being compared against the individuals target training HR as described above.

Execution of the IE training protocol in the home will be assessed for each individual participant at the end of the first week by checking to see if their mean training heart rate per week falls within target heart rate range / reference interval (76-111\%) data for weeks 4, 12 and 24 will now be assessed at the end of the 6-month training period. An estimate of the short- (4-week) and medium-term (3- and 6-month) adherence rates to IE training will be based upon the data collected from participant diaries to calculate the proportion of participants completing $\geq$ two thirds of all IE sessions (12, 36 and 72) at each time point respectively. Patient recruitment and participant attrition rates will be calculated based upon the average number of participants recruited per week over the 7-month recruitment period and the number of withdrawals from the study once the last follow-up call to the last participant has been made.

Participant experiences of IE will be assessed through a quantitative online survey conducted at week 4. All participants receiving the IE intervention will be invited to take part in one of two focus groups (remote) to draw out respondents' attitudes, feelings, beliefs and experiences regarding the intervention (40). This will also be used as an opportunity to explore possible negative effects of COVID-19 on recruitment rates and participation. These groups will be held at month 4 and month 8 of the recruitment period with 6-8 participants in each group. One focus group will also be undertaken with healthcare professionals involved in the intervention delivery at the end of the recruitment period to explore views and experiences of the IE intervention. Lay and professional members of the research team will coproduce the topic guide and co-facilitate the focus groups. The focus groups are proposed to last for between 60-90 minutes. The groups will be digitally recorded and transcribed. Telephone interviews $(n=5-$ 10) will be conducted with stakeholders from GP practices which are not recruitment sites and not involved in the intervention delivery to explore the willingness of GPs and primary care healthcare professionals to consider IE as a viable treatment option for patients, including barriers and facilitators for delivering and integrating this within an NHS care pathway for hypertension.

The economic evaluation of delivering the IE intervention will be calculated once the last follow-up call has been made to the last participant and will be estimated from Healthcare resource use data and 
quality-adjusted life years (QALYs). Since QALYs are the primary outcome of the economic evaluation, utility values will be obtained from patients' responses to each of the EQ-5D-5L at the beginning of the intervention and at week 4, and months 3 and 6 after the intervention (41-42).

New insight into the accuracy of home BP measurements to monitor changes in BP will be based upon the BP data (observed and home readings) recorded (at Baseline Assessment - Day 1, Assessment 2 Week 4, Assessment 3 - Month 3 and Assessment 4 - Month 6) in the participant diaries; along with expert evaluation of the participants ability to carry out the measure.

\section{Plans to promote participant retention and complete follow-up $\{18 \mathrm{~b}\}$}

Participant adherence will be measured as outcome data including the number of IE sessions completed. Completion of at least 8 of 12 sessions between baseline and the 4-week timepoint will be deemed adherence to the intervention. The percentage of completed IE sessions that meet the required target HR threshold will be calculated. The percentage of participants that deviate from protocol will be recorded to assess fidelity to the IE programme. The rate of healthcare professionals that pass the competency assessment after the half-day training session will be calculated.

\section{Data management $\{19\}$}

Data entered directly into paper case report forms is considered as source data, additional source documentation includes participant study diaries, online questionnaires and focus group/ interview audio recordings and transcripts. Data from case report forms and participant diaries will be entered manually into the database allowing for data monitoring and cleansing. Questionnaire data will be received and directly accessible online by only research team members who have the appropriate access. Case report forms will be shared with the coordinating center via password secured email and a copy retained securely at site. A unique code will be produced for each participant and used on all corresponding documentation and files, to ensure anonymity.

\section{Confidentiality $\{27\}$}

Only anonymised data will be shared with the coordinating centre for analysis. Electronic files with personal information will be password protected and stored on the university partner networks in folders that can only be accessed by the research team. Access to the data collected during the project (including any participant personal data) will be restricted to the research team, and data will not be shared with anyone else. Personal information that may enable the service user to be identified will be removed from interview and focus group transcripts.

Any personal data will be destroyed on completion of the project. The coded data will be stored for five years following the completion of the study, when it will be destroyed.

Plans for collection, laboratory evaluation and storage of biological specimens for genetic or molecular analysis in this trial/future use $\{33\}$ 
Not applicable

\section{Statistical methods}

\section{Statistical methods for primary and secondary outcomes $\{20 \mathrm{a}\}$}

\section{Quantitative data analysis}

Descriptive statistics will be used to assess primary and secondary process outcomes such as recruitment rates, adherence rates and completeness of data. Exercise adherence will be compared with outcomes to inform compliance criteria in the full study. In a definitive study, the primary outcome change from baseline in systolic BP - will be analysed using analysis of covariance (ANCOVA), with a fixed treatment effect allowing adjustments for baseline values, centre, sex and age. This model will be used to estimate differences between the arms and confidence intervals from the feasibility study. $80 \%$ and $95 \%$ confidence intervals will be calculated. Data from the IE experience questionnaires will be transferred to SPSS (v24) and analysed using descriptive statistics.

\section{Qualitative data analysis}

Thematic analysis of focus group/interview transcripts will be carried out using Braun and Clarke's (2013) six stage model using NVIVO v11 (43). Drawing on Sweeney et al.'s (44) notion that the service user researcher unique perspective should be preserved rather than subsumed, the process will involve multiple members (including public co-applicants) of the project team. Inductive thematic analysis of focus group/interview transcripts will be carried out using NVIVO 11. The process will involve reading and re-reading the transcripts and noting down initial ideas. Then the transcripts will be coded. Data extracts will be collated within each code and then codes ordered into potential themes. Subsequently, these themes will then be reviewed and refined. Ongoing analysis will refine the specifics of each theme and identify any themes which have not previously been recognised. Deviant case analysis will be used to ensure that perspectives that diverged from dominant trends are not overlooked.

\section{Interim analyses $\{21 b\}$}

Not applicable

Methods for additional analyses (e.g. subgroup analyses) \{20b\}

Not applicable

Methods in analysis to handle protocol non-adherence and any statistical methods to handle missing data $\{20 \mathrm{c}\}$

Baseline observation carried forward will be used in the secondary analysis of intention to treat.

Plans to give access to the full protocol, participant level-data and statistical code $\{31 \mathrm{c}\}$ 
Anonymised data will be made available to researchers at universities, NHS organisations or other healthcare providers where the sharing of data has a clear defined purpose and its use will be of benefit to wider society.

\section{Oversight and monitoring}

\section{Composition of the coordinating centre and trial steering committee $\{5 \mathrm{~d}\}$}

Delivery of the project is a collaboration between the Section of Sport, Exercise \& Rehabilitation Sciences, Canterbury Christ Church University (CCCU), the Centre for Health Services Studies, University of Kent (UKC) and East Kent Hospitals University Foundation Trust (EKHUFT).

Two groups were established to provide appropriate oversight of the study and ensure this is maintained from various perspectives, the Study Steering Committee (SSC) and the Project Management Group (PMG).

The SSC is comprised of at least $75 \%$ independent members and includes the project co-leads, an independent statistician, a health economist, a clinician with expertise in hypertension/clinical trials/primary care, an exercise specialist and two independent lay members. The SSC meets tri-monthly to: critically oversee progress, outputs, deliverables and governance of the project; have oversight of delivery of the study on behalf of the funder to ensure achievement of study objectives within agreed timelines; ensure the protection of rights and safety of study participants; regularly review of ongoing project data; periodically review of safety data to ensure patient safety throughout (45). In doing so, the SSC reports directly to the funder. The SSC will consider the need for any interim analysis based on reports received and may consider dat emerging from other related studies and make recommendations for this study based on these. The SSC will also consider whether further time or funding is required for any aspect of the study and advise where best this may be obtained.

The PMG is comprised of 2 co-chief investigators, 4 co-applicants (including the project manager), 1 statistician, 1 health economist, 2 public co-applicants, 1 research facilitator and 1 study coordinator (45). The members of PMG were responsible for the elaboration of this protocol and meets fortnightly with the purpose of: maintaining clear oversight of study delivery according to the protocol, original grant application and subsequent changes; assessing the progress of the study and identifying any barriers to completion; agreeing mitigation plans and actions for any barriers to study completion e.g. the current Covid-19 pandemic; providing advice to the Chief Investigators; ensuring the protection of rights and safety of study participants; and reporting on study progress to the SSC and funder.

The organizational study chart will include the sponsor EKHUFT, the SSC, the PMG, the two research units; UKC as lead on Qualitative data (health economics, focus group, interviews, patient participant involvement etc.) and CCCU as lead on quantitative data (intervention delivery, fidelity and patient outcomes etc.), the clinical research lead and the investigators at each primary care recruitment sites in SE England. 
Composition of the data monitoring committee, its role and reporting structure $\{21 \mathrm{a}\}$

A data monitoring committee is not needed for this study because the intervention is non-invasive with minimal risk of harm.

\section{Adverse event reporting and harms $\{22\}$}

Only adverse events (AE) resulting (definitely or probably) from study procedures or the intervention will be collected by the research team and recorded in the participant's medical records. All serious adverse events (SAEs) will be recorded within 24 hours of knowledge of the event. The Chief investigator will report all SAEs to the Sponsor and the Research Ethics Committee within the conditions of ethical approval. The Study Steering Committee will periodically review safety data to ensure patient safety throughout.

\section{Frequency and plans for auditing trial conduct $\{23\}$}

Regular monitoring will be performed by the Study Coordinator to evaluate compliance with the protocol. Monitoring will verify that the study is conducted, and data are generated, documented and reported in compliance with the protocol and the applicable regulatory and national policy requirements (45). Any data issues will be addressed by raising data queries for GP sites to resolve where possible, following this on-site monitoring will be undertaken. Direct access will be granted to authorised representatives from the Sponsor, host institution and the regulatory authorities to permit trial-related monitoring, audits and inspections- in line with participant consent.

\section{Plans for communicating important protocol amendments to relevant parties (e.g. trial participants, ethical committees) $\{25\}$}

Any substantial amendments to the protocol or other study documents may require review and approval by the REC before the changes can be implemented to the study. Where amendments are required, NHS HRA and REC procedures will be followed.

\section{Dissemination plans $\{31$ a $\}$}

Results will be presented at national and international conferences (e.g. UKactive Summit) and findings published in high-impact, open access peer-reviewed journals in the field where the project team's previous work has been well received (e.g. Hypertension, Journal of Human Hypertension, Journal of Hypertension, Journal of Applied Physiology, PLOS Medicine, British Medical Journal).

Study participants will receive a personalised report of their own results and a summary of study findings will also be sent to GP practices and local organisations (E.g. Canterbury and Costal Clinical Commissioning Group, EKHUFT, Kent and Medway STP, Kent AHSN and NIHR CRN:KSS). The study final report will be submitted and a lay summary disseminated to a wider audience to create national impact by engaging with policy and national governing body organisations (e.g., Public Health England, The 
National Institute for Health and Care Excellence, National Centre for Sport \& Exercise Medicine), third sector organisations (e.g. British Heart Foundation, British Hypertension Society) with the aim of understanding the evidence required to influence current hypertension treatment guidelines with respect to offering lifestyle interventions like IE as standard care and a tangible product that can be marketed in the NHS.

\section{Discussion}

Systematic isometric wall squat exercise, which employs the large muscle mass of the quadriceps, has been shown to be an effective means of lowering resting BP. In a recent relatively small-scale study, Taylor et al. ${ }^{19}$ showed that the magnitude of BP reduction following 4 weeks of isometric wall squat exercise in unmedicated high normal participants was greater than when the same intervention was completed by those with BP in the normal range ${ }^{38}$. It was postulated that this IE intervention may exert a greater antihypertensive effect in patients with more severe hypertension such as those diagnosed with Stage 1 hypertension. The clinically significant BP reduction observed was also greater than the average $\mathrm{BP}$ reduction achieved with a single, standard dose anti-hypertensive drug (46) and is associated with reduced cardiovascular mortality (30). However, before the efficacy of this novel intervention to treat Stage 1 hypertension can be investigated in any large-scale randomised controlled trial, it is first necessary to ascertain if it can be delivered and carried out as intended in (as ultimately envisioned) an NHS primary care setting. If it can, and patient adherence is found to be equal or better than that documented for other forms of lifestyle invention and resting BP is shown to either decrease or stay the same following the IE intervention, then the findings of this feasibility study will support the viability of IE as both a prophylactic and alternative treatment option for thousands of NHS patients wishing to avoid antihypertensive drug therapy. Importantly the results of this study will determine uncertain parameters needed to design a substantive efficacy study, including the variance in BP change needed for sample size calculation, the ability to deliver the intervention as intended in primary care, recruitment rates, and participant acceptability and compliance. Moreover, the economic analysis in this feasibility study will determine the acceptability of resource use and utility measures in this setting, establish the cost of the IE intervention programme and conduct some preliminary modelling to explore potential cost effectiveness, prior to a full economic evaluation of the IE intervention programme in the planned definitive trial. The follow-on study will be a randomised, controlled efficacy study pending additional funding. This study will determine uncertain parameters needed to design a substantive trial; variance of the primary outcome measure needed for sample size calculation, ability to deliver the intervention as intended in primary care, recruitment rates and participant acceptability and compliance.

Whilst the advent of Covid-19 has presented a significant challenge, primarily in terms of reliably delivering and evaluating a novel exercise training intervention remotely, it has also provided a valuable opportunity to evaluate alternate methodological approaches / new protocols that may not otherwise have occurred, e.g. the feasibility of using observed patient home BP readings for remote monitoring as opposed to clinic measurements. Since the current pandemic shows little sign of abating, this additional 
information might help to inform the research design of similar studies planned for delivery during these exceptional times.

\section{Trial Status}

The protocol reported here is version 3.2.1 and was last updated on 1st October 2020. The target date to start recruitment is 18th January 2021 and based upon a predicted 7 months for recruitment, this will be completed by 31st August 2021.

\section{Abbreviations}

BP: blood pressure; IE: isometric exercise; NHS: National Health Service; PHE: Public Health England; CKDEPI: Chronic Kidney Disease Epidemiology Collaboration; MDRD: Modification of Diet in Renal Disease; QALYs: quality-adjusted life years; AE: adverse event; SAE: serious adverse event; CCCU: Canterbury Christ Church University; UKC: University of Kent; EKHUFT: East Kent Hospitals University Foundation Trust; SSC: Study Steering Committee; PMG: Project Management Group.

\section{Declarations}

\section{Acknowledgements}

Not applicable

\section{Authors' contributions \{31b\}}

JW led the writing of this study protocol submission, and all other authors (all initials) contributed equally with comments and feedback. All authors read and approved the final manuscript.

\section{Funding $\{4\}$}

The National institute for Health Research (NIHR) Research for Patient Benefit (RfPB) funded the study.

\section{Availability of data and materials $\{29\}$}

Anonymised data will be made available upon request, to researchers at universities, NHS organisations or other healthcare providers where the sharing of data has a clear defined purpose and its use will be of benefit to wider society.

\section{Ethics approval and consent to participate $\{24\}$}

The project was approved by an National Health Service (NHS) and Heath Research Authority (HRA) Ethics Committee on 22 ${ }^{\text {nd }}$ May 2020. Research Ethics Committee number 20/LO/0422. Participants will be enrolled to the study once they have given informed consent. 
NIHR

\section{Competing interests $\{28\}$}

The authors declare that they have no competing interests

\section{Authors' information (optional)}

${ }^{1}$ Faculty of Science, Engineering and Social Sciences, Canterbury Christ Church University, Canterbury, Kent UK. ${ }^{2}$ Centre for Health Services Studies, University of Kent, Canterbury, Kent, UK. ${ }^{3}$ Renal Department East Kent Hospitals University NHS Foundation Trust, Canterbury, Kent, UK. ${ }^{4}$ Newton Place Surgery, Faversham, Kent, UK. ${ }^{5}$ Public co-applicant, Kent, UK.

\section{References}

1. The National Institute for Health and Care Excellence (NICE). Hypertension in adults: diagnosis and management 2019. https://www.nice.org.uk/guidance/ng136 (2019). Accessed 19 Nov 2020.

2. Lim SS, Vos T, Flaxman AD, Danaei G, Shibuya K, Adair-Rohani H, et al. A comparative risk assessment of burden of disease and injury attributable to 67 risk factors and risk factor clusters in 21 regions, 1990- 2010: a systematic analysis for the Global Burden of Disease Study 2010. Lancet. 2012. doi:https://doi.org/10.1016/S0140-6736(12)61766-8.

3. Public Health England. Tackling high blood pressure. From evidence into action. https://www.gov.uk/government/publications/high-blood-pressure-action-plan (2014). Accessed 19 Nov 2020.

4. Wilkins E, Wilson L, Wickramasinghe K, Bhatnagar P, Leal J, Luengo-Fernandez R, et al. European Cardiovascular Disease Statistics 2017 Edition. In: European Heart Network. 2017. http://www.ehnheart.org/cvd-statistics/cvd-statistics-2017.html. Accessed 19 Nov 2020.

5. Mancia G. The association of hypertension and diabetes: prevalence, cardiovascular risk and protection by BP reduction. Acta diabetologica. 2005. doi:https://doi.org/10.1007/s00592-005-0177z.

6. Corrao G, Zambon A, Parodi A, Poluzzi E, Baldi I, Merlino L, et al. Discontinuation of and changes in drug therapy for hypertension among newly-treated patients: a population-based study in Italy. $\mathrm{J}$ Hypertens. 2008. doi:https://doi.org/10.1097/hjh.0b013e3282f4edd7.

7. Chobanian AV, Bakris GL, Black HR, Cushman WC, Green LA, Izzo JL, et al. The Seventh Report of the Joint National Committee on Prevention, Detection, Evaluation, and Treatment of High BP: the JNC 7 report. Jama. 2003. doi:https://doi.org/10.1001/jama.289.19.2560.

8. Sanderson J, Kay N, Watts R. Universal Personalised Care Implementing the Comprehensive Model. In: NHS England. 2019. https://www.england.nhs.uk/wp-content/uploads/2019/01/universalpersonalised-care.pdf. Accessed Jan 2019. 
9. Gao SK, Fitzpatrick AL, Psaty B, Jiang R, Post W, Cutler J, et al. Suboptimal nutritional intake for hypertension control in 4 ethnic groups. Arch Intern Med. 2009; 169(7):702-7. doi:

10.1001/archinternmed.2009.17. PMID: 19365000.

10. Pescatello LS, Franklin BA, Fagard R, Farquhar WB, Kellley GA, Ray CA. American College of Sports Medicine. American College of Sports Medicine position stand. Exercise and hypertension. Med Sci Sports Exerc. 2004. doi:https://doi.org/10.1249/01.mss.0000115224.88514.3a.

11. Cornelissen VA, Smart NA. Exercise training for BP: a systematic review and meta-analysis. J Am Heart Assoc. 2013. doi:https://doi.org/10.1161/JAHA.112.004473.

12. Owen A, Wiles J, Swaine I. Effect of isometric exercise on resting blood pressure: a meta-analysis. J Hum Hypertens. 2010. doi:https://doi.org/10.1038/jhh.2010.13.

13. Zaleski A. Exercise for the Prevention and Treatment of Hypertension - Implications and Application. In: American College of Sports Medicine. https://www.acsm.org/blog-detail/acsm-certifiedblog/2019/02/27/exercise-hypertension-prevention-treatment (2019). Accessed 03 Aug 2020.

14. Blumenthal JA, Siegel WC, Appelbaum M. Failure of exercise to reduce BP in patients with mild hypertension. Results of a randomized controlled trial. JAMA. 1991;266(15):2098-104. doi:10.1001/jama.1991.03470150070033.

15. Linke SE, Gallo LC, Norman GJ. Attrition and adherence rates of sustained vs. intermittent exercise interventions. Ann Behav Med. 2011;42(2):197-209. doi: https://dx.doi.org/10.1007\%2Fs12160-0119279-8.

16. Gordon BDH, et al. A Comparison of BP Reductions Following 12-Weeks of Isometric Exercise Training Either In The Laboratory Or At Home. Journal of the American Society of Hypertension. 2018. doi:https://doi.org/10.1016/j.jash.2018.09.003.

17. Carlson DJ, Dieberg G, Hess NC, Millar PJ, Smart NA. Isometric exercise training for BP management: A systematic review and meta-analysis. Mayo Clin Proc. 2014; doi: https://doi.org/10.1016/j.mayocp.2013.10.030.

18. Wiles JD, Goldring N, Coleman D. Home-based isometric exercise training induced reductions resting blood pressure. Euro j of app physiol. 2017. doi:https://doi.org/10.1007/s00421-016-3501-0.

19. Taylor KA, et al. Neurohumoral and ambulatory haemodynamic adaptations following isometric exercise training in unmedicated hypertensive patients. $\mathrm{J}$ of Hypertens. 2018. doi:https://doi.org/10.1097/hjh.0000000000001922.

20. NHS launches new drive to save thousands of people from heart attacks and strokes. NHS news 2017. https://www.england.nhs.uk/2017/09/nhs-launches-new-drive-to-save-thousands-of-peoplefrom-heart-attacks-and-strokes/ (2017). Accessed Aug 2020.

21. Wiles JD, Coleman DA, Swaine IL. The effects of performing isometric training at two exercise intensities in healthy young males. European journal of applied physiology. 2010. doi:https://doi.org/10.1007/s00421-009-1025-6.

22. Millar PJ, McGowan CL, Cornelissen VA, Araujo CG, Swaine IL. Evidence for the role of isometric exercise training in reducing BP: potential mechanisms and future directions. Sports med. 2014. 
doi:https://doi.org/10.1007/s40279-013-0118-x.

23. O'Driscoll JM, Taylor KA, Wiles JD, Coleman DA, Sharma R. Acute cardiac functional and mechanical responses to isometric exercise in prehypertensive males. Physiological reports. 2017; doi: https://dx.doi.org/10.14814\%2Fphy2.13236.

24. Devereux GR, Wiles JD, Swaine IL. Reductions in resting BP after 4 weeks of isometric exercise training. European journal of applied physiology. 2010. doi:https://doi.org/10.1007/s00421-0101394-x.

25. Howden R, Lightfoot T, Brown SJ, Swaine IL. The effects of isometric exercise training on resting BP and orthostatic tolerance in humans. Exp Physiol. 2002. doi:https://doi.org/10.1111/j.1469445x.2002.tb00064.x.

26. Goldring N, Wiles JD, Coleman D. The effects of isometric wall squat exercise on heart rate and BP in a normotensive population. Journal of Sports Sci. 2014. doi:https://doi.org/10.1080/02640414.2013.809471.

27. Millar PJ, MacDonald MJ, Bray SR, McCartney N. Isometric handgrip exercise improves acute neurocardiac regulation. European journal of applied physiology. 2009. doi:https://doi.org/10.1007/s00421-009-1142-2.

28. Taylor KA, Wiles JD, Coleman D, Sharma R, O’Driscoll JM. Continuous cardiac autonomic and haemodynamic responses to isometric exercise. Medicine Science in Sport Exercise. 2017. doi:https://doi.org/10.1249/mss.0000000000001271.

29. Millar PJ, Levy AS, McGowan CL, McCartney N, MacDonald MJ. Isometric handgrip training lowers BP and increases heart rate complexity in medicated hypertensive patients. Scand J Med Sci Sports. 2013. doi:https://doi.org/10.1111/j.1600-0838.2011.01435.x.

30. Prospective Studies Collaboration. Age-specific relevance of usual blood pressure to vascular mortality: a meta-analysis of individual data for one million adults in 61 prospective studies. Lancet. 2002. doi:https://doi.org/10.1016/S0140-6736(02)11911-8.

31. Lascar N, Kennedy A, Hancock B, Jenkins D, Andrews RC, Greenfield, et al. Attitudes and Barriers to Exercise in Adults with Type 1 Diabetes (T1DM) and How Best to Address Them: A Qualitative Study. PLOS ONE. 2014. doi:https://doi.org/10.1371/journal.pone.0108019.

32. NHS. NHS exercise recommendations for adults- Exercise. https://www.nhs.uk/live-well/exercise/. (2019) Date accessed Aug 2020.

33. Jakicic JM, Wing RR, Buler BA, Robertson RJ. Prescribing exercise in multiple short bouts versus one continuous bout: effects on adherence, cardiorespiratory fitness, and weight loss in overweight women. International journal of obesity related metabolic disorders: J Intern Assoc for the Study of Obesity. 1995;19(12):983-01.

34. Poltawski L, Boddy K, Forster A, Goodwin VA, Pavey AC, Dean S. Motivators for uptake and maintenance of exercise: perceptions of long-term stroke survivors and implications for design of exercise programmes. Disabil Rehab. 2014;37(9):795-801.

doi:https://doi.org/10.3109/09638288.2014.946154. 
35. World Health Organisation Regional Office for Europe. (2020): Coronavirus disease (COVID-19) pandemic. https://www.euro.who.int/en/health-topics/health-emergencies/coronavirus-covid19/novel-coronavirus-2019-ncov (2020). Accessed 28 Aug 2020.

36. National Institute of Health Research. Restart Framework. https://www.nihr.ac.uk/documents/restartframework/24886?pr (2020). Accessed 19 Nov 2020.

37. Wiles JD, Goldring N, O'Driscoll JM, Taylor KA, Coleman DA. An alternative approach to isometric exercise training prescription for cardiovascular health. Translational Journal of the ACSM. 2018;3(2):10-8. doi:https://doi.org/10.1249/TJX.0000000000000052.

38. Teare MD, Dimairo M, Shephard N, Hayman A, Whitehead A, Walters J. Sample size requirements to estimate key design parameters from external pilot randomised controlled trials: a simulation study. Trials. 2014 Jul 3;15:264. doi: https://doi.org/10.1186/1745-6215-15-264.

39. Sealed envelope. Randomisation and online databases for clinical trials. https://www.sealedenvelope.com/ (2020). Accessed Aug 2020.

40. Krueger R, Casey MA. Focus Groups: A Practical Guide for Applied Research. 3rd ed. Thousand Oaks: Sage Publications; 2000.

41. NICE. Position statement on use of the EQ-5D-5L valuation set for England (updated. November 2018). https://www.nice.org.uk/about/what-we-do/our-programmes/nice-guidance/technologyappraisal-guidance/eq-5d-5l. (2018) Accessed Aug 2020.

42. Van Hout B, Janssen MF, Feng Y, Kohlmann T, Busschbach J, Golicki D, et al. Interim scoring for the EQ-5D-5L: Mapping the EQ-5D-5L to EQ-5D-3Lvalue sets. Value in Health. 2012;15:708-15. doi:https://doi.org/10.1016/j.jval.2012.02.008.

43. Braun V, Clarke V. Successful Qualitative Research: A Practical Guide for Beginners. London: Sage; 2013.

44. Sweeney A, Greenwood K, Williams S, Wykes T, Rose D. Hearing the voices of service user researchers in collaborative qualitative data analysis: the case for multiple coding. Health Expect. 2013. doi:10.1111/j.1369-7625.2012.00810.x.

45. The UK policy framework for health and social care research. The Health Research Authority. https://www.hra.nhs.uk/planning-and-improving-research/policies-standards-legislation/uk-policyframework-health-social-care-research/. (2020) Accessed Aug 2020.

46. Law MR, Morris JK, Wald NJ. Use of blood pressure lowering drugs in the prevention of cardiovascular disease: Meta-analysis of 147 randomised trials in the context of expectations from prospective epidemiological studies. BMJ. 2009;338:b1665.

\section{Figures}



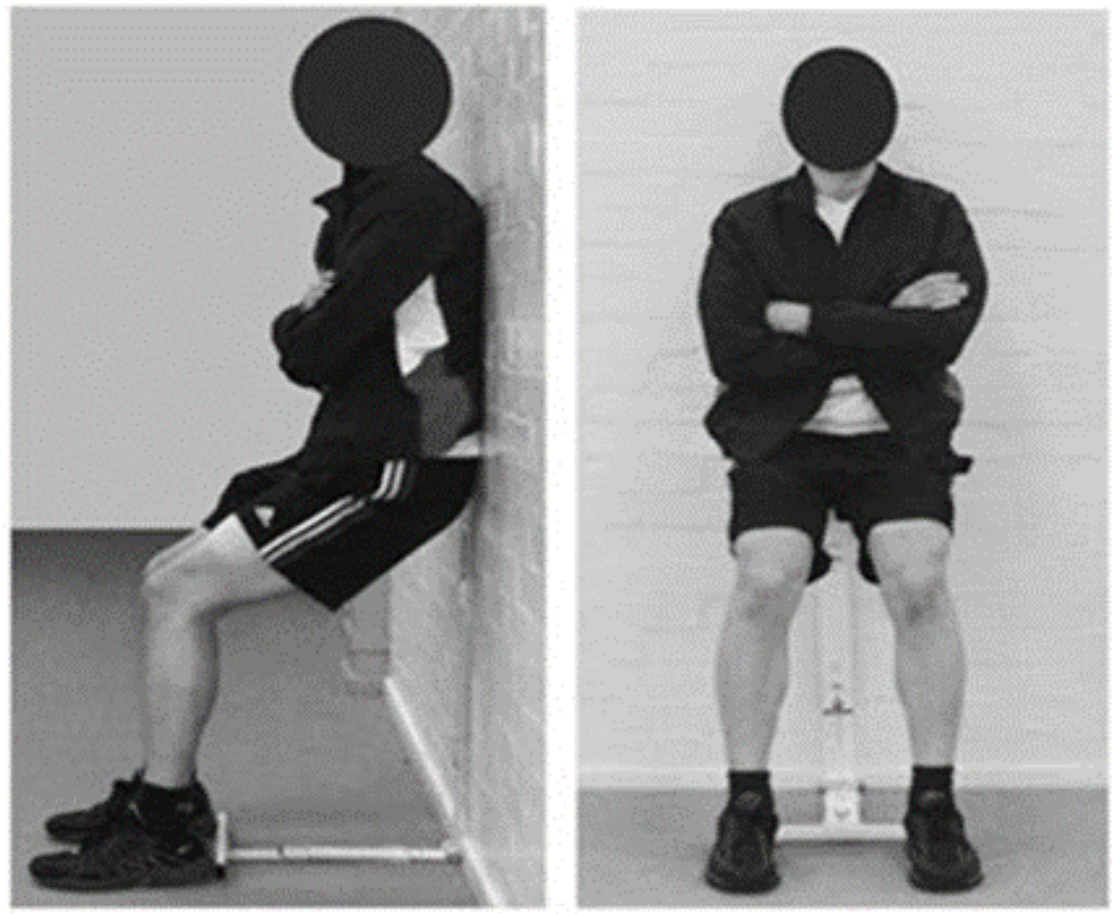

Figure 1

Isometric wall squat exercise 


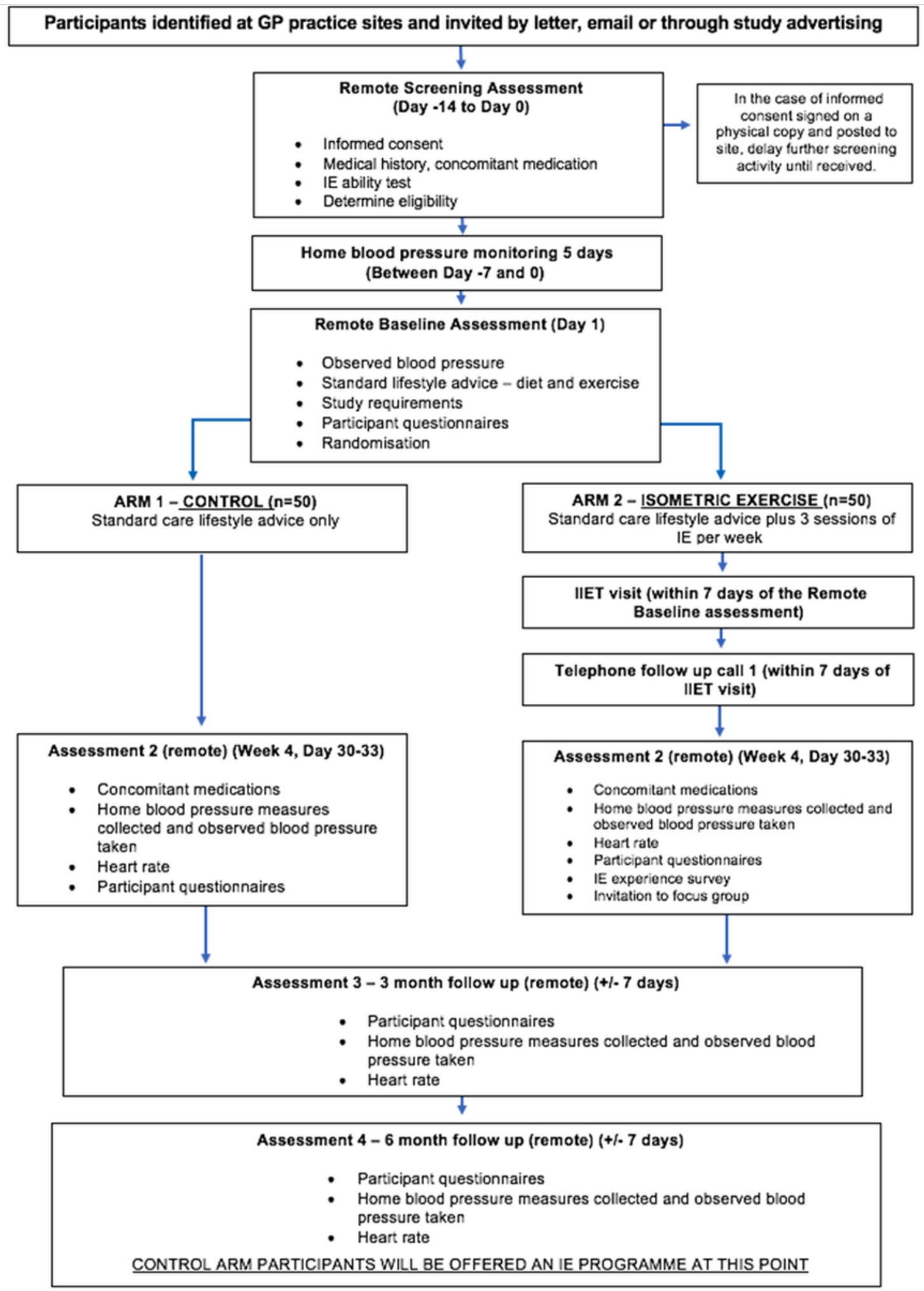

\section{Figure 2}

Trial Flow chart 